\title{
Development of High Throughput Cryo Electron Microscope with Cold Field Emission Gun (CRYO ARM ${ }^{\mathrm{TM}} 300$ II)
}

AKIHIRO OOSAKI, Naoki Hosogi, Fumiaki Makino, Sohei Motoki, Isamu Ishikawa, Yoshihiro Ookura and Kazumi Kobayashi

JEOL EM Technical Development Department, Higashiyamatoshi, Japan

[Outline]

$\mathrm{X}$-ray diffraction and nuclear magnetic resonance have been used for the structural analysis of the biomolecules such as proteins. In recent years, a structural analysis by Single-Particle Analysis (SPA), using a cryo electron microscope, has been getting popular. The reasons for this are that the method requires no crystal samples and that the method gives drastically improved spatial resolution. It was realized by new analysis software and a direct detection high speed camera. And the SPA requires large number of images for obtaining good reconstruction. Therefore, these images should be collected by automatic acquisition system. The number of these images is essentially indispensable for improvement of the signal to noise ratio $(\mathrm{S} / \mathrm{N})$ by averaging in image processing. Quite recently, to meet these requirements, we developed a cryo TEM (CRYO ARM ${ }^{\mathrm{TM}} 300$ II shown in Fig. 1) better than the previous equipment on data throughput and usability. These improvements were realized by the new functions shown as follows.

[New function]

1. Quick Collection of images

The new machine provides highly stable beam-shifting system accompanied with electro magnetic image shifting system. The key feature of the system is high beam stability shown in Fig. 2. This system provides quick image acquisition with no sample stage move.

\section{Zero Fringe illumination System (ZFS}

The machine provides an unique "Koehler mode" illumination beam, which has no interference fringes. This eliminates unusable illuminated area with the fringes, resulting in less electron-beam damage.

\section{NEW CFEG}

The microscope is equipped with a cold field emission electron gun (CFEG), which provides a low energy spread and an improved probe current stability, allowing users to obtain high quality images at any time.

4. NEW omega filter with auto tuning system

The machine is equipped with a new improved in-column Omega type energy filter with auto tuning system. The system provides self-adjusting and always delivers the best performance with high stability. The auto tuning system is built into the operating software "TEM Center" and operable from TEM ordinal graphic user interface.

\section{High precision stage drive}


The new machine has a rotation free stage transfer system. The advantage of this stage is excellent positional reproducibility. Even if samples are transferred back and forth between the microscope column and sample storage, the low magnification images, which we call "global map", can be shared.

\section{[Result]}

As a result, we became able to collect 22,000 images/day by introducing these new functions. And we have succeeded to perform SPA with short time.

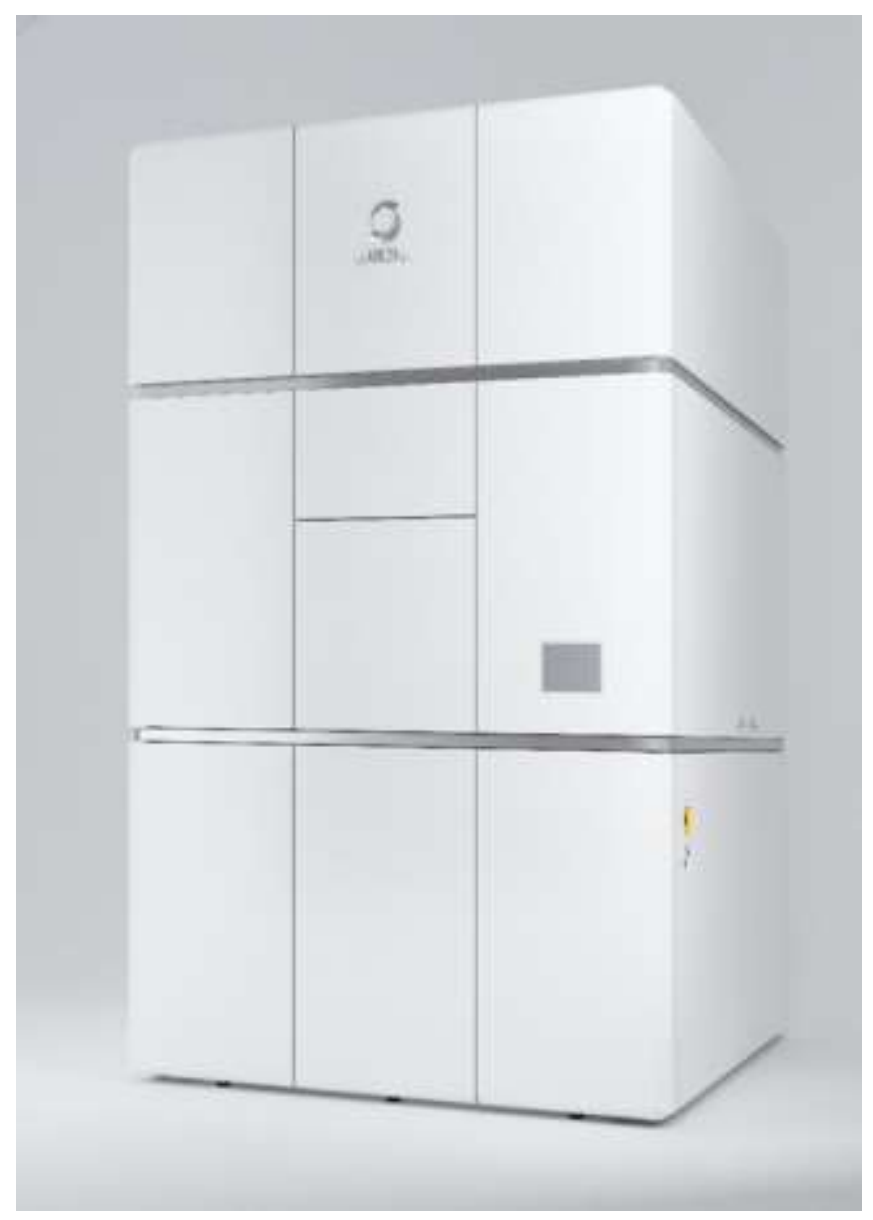

Figure 1. Appearance of the new cryo microscope (CRYO ARM $\left.{ }^{\mathrm{TM}} 300 \mathrm{II}\right)$. This cryo-electron microscope efficiently and quickly produces images of electron beam-sensitive specimens, for single particle analysis, tomography and MicroED. This microscope system offers improved throughput, easeof-use and stability, compared to the cryo-TEMs of previous generation. 


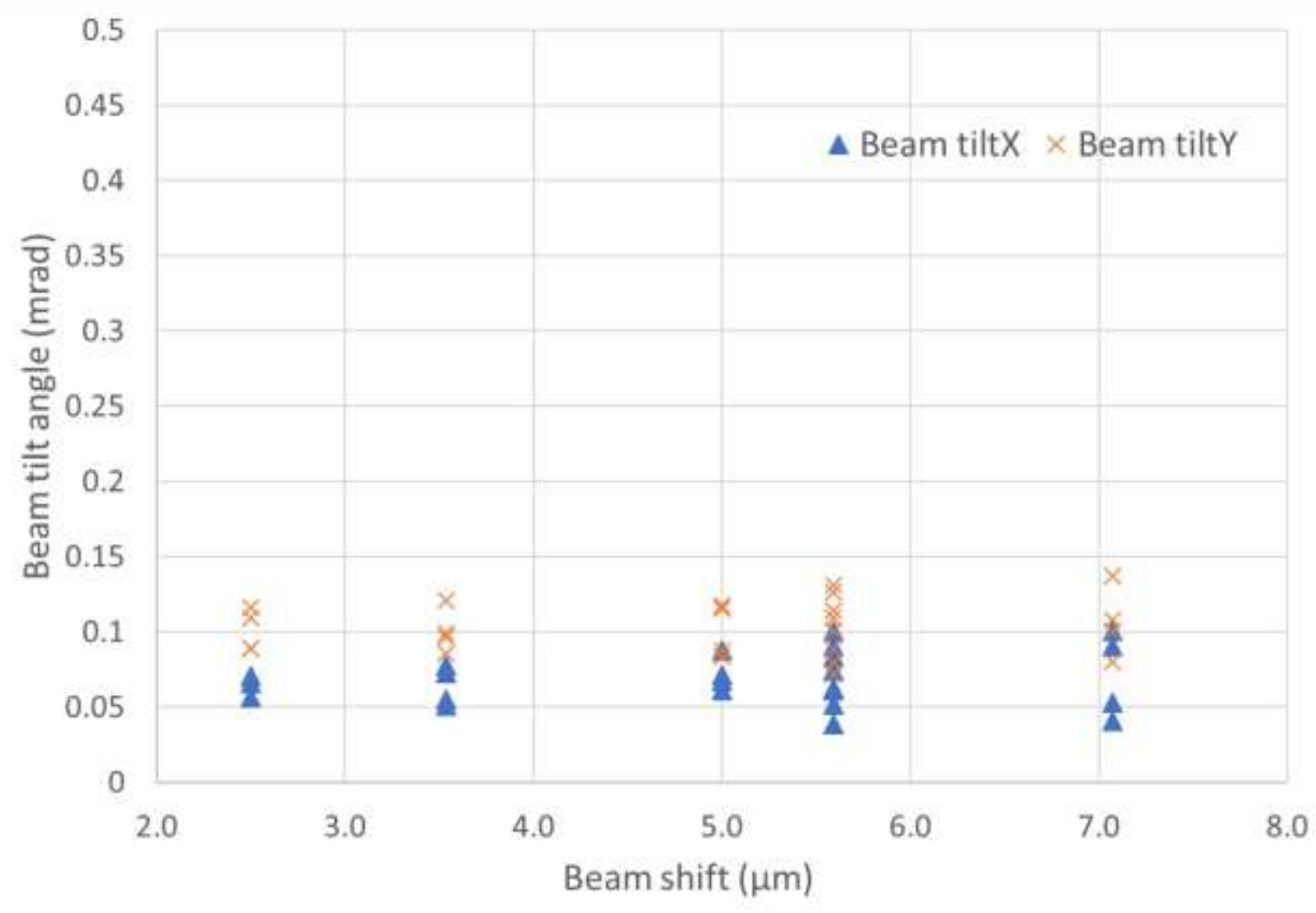

Figure 2. This figure shows beam tilt, when the Koeler-illuminated beam was shifted by several micrometers. The beam tilts were measured to be less than $0.15 \mathrm{mrad}$ even if the beam shifts by $7.1 \mu \mathrm{m}$. Thus, we could minimize the influence of coma aberration in acquired images. 